\title{
Mycotic Aneurysms: Clinical Aspects and Results of Surgery (About 12 Cases)
}

\author{
Momar Sokhna Diop*, Ndeye Fatou Sow, Pape Ousmane Ba, Magaye Gaye, Papa Amath Diagne, \\ Pape Adama Dieng, Souleymane Diatta, Pape Salmane Ba, Moussa Seck Diop, \\ Mareme Soda Mbaye, Amadou Gabriel Ciss, Assane Ndiaye, Mouhamadou Ndiaye
}

Department of Cardiovascular and Thoracic Surgery, Cheikh Anta Diop University, Dakar, Senegal

\section{Email address:}

momarsokhna08@yahoo.fr (M. S. Diop),zenefes@gmail.com (N. F. Sow), papy.ba@gmail.com (P. O. Ba), mgaye99@yahoo.fr (M. Gaye), diagnepapaamathdiagne@gmail.com (P. A. Diagne), padiengsala@yahoo.fr (P. A. Dieng), soul.diatta@yahoo.fr (S. Diatta), mansalb@yahoo.fr (P. S. Ba), sirmsd9@gmail.com (M. S. Diop), sodaabeck24@yahoo.fr (M. S. Mbaye), cissgaby@yahoo.fr (A. G. Ciss), lazanat@yahoo.fr (A. Ndiaye), mondiaye@orange.sn (M. Ndiaye)

${ }^{*}$ Corresponding author

\section{To cite this article:}

Momar Sokhna Diop, Ndeye Fatou Sow, Pape Ousmane Ba, Magaye Gaye, Papa Amath Diagne, Pape Adama Dieng, Souleymane Diatta, Pape Salmane Ba, Moussa Seck Diop, Mareme Soda Mbaye, Amadou Gabriel Ciss, Assane Ndiaye, Mouhamadou Ndiaye. Mycotic Aneurysms: Clinical Aspects and Results of Surgery (About 12 Cases). Cardiology and Cardiovascular Research. Vol. 3, No. 1, 2019, pp. 1-5. doi: 10.11648/j.ccr.20190301.11

Received: October 23, 2018; Accepted: December 6, 2018; Published: January 24, 2019

\begin{abstract}
Introduction: Mycotic aneurysms have an incidence of 1 to $2 \%$. Before the era of antibiotics, syphilis was most commonly observed. However, with the increase in arterial interventional procedures and intravenous drug use, Gram-positive organisms such as Staphylococcus and Streptococcus are observed. Objectives: To analyze the clinical aspects and to evaluate the results of the surgery. Materials and Methods: This was a descriptive retrospective study over a 12-years period from December 2005 to February 2018. Results: The total number of our series was 12 cases. The average age at the time of surgery was 37 years old [17-83 years]. There was no predominance of sex. There was a notion of smoking in 3 cases / 12. There was no concept of addiction. Four out of 12 cases had a known concept of heart disease with mitral insufficiency, aortic insufficiency, and complete atrioventricular block. A patient presented in this antecedents a notion of syphilis treated and declared cured. The clinical symptomatology was made of fever in 4 cases out of 12, pain in 11 cases out of 12 with 3 cases of intermittent claudication. The clinical examination had shown a swelling with vascular characters in 11 cases out of 12 . Two out of 12 patients had signs of acute limb ischemia. The arterial echodoppler was performed in 11 cases out of 12 which had made the diagnosis. The angioscan was performed in 8 cases and showed 4 cases of sacciform aneurysms. All patients benefited from open surgery. The exploration showed 9 cases of false aneurysms with signs of local infection in 5 cases. Flattening with excision of the infected tissues was performed in all cases. The restoration of vascular continuity was immediate in all cases by end-to-end direct anastomosis in 8 cases, 2 cases of extra-anatomical bypass using a dacron tube and two cases of anatomical bypass using the saphenous vein in situ. Early complications were dominated by 2 cases of acute limb ischemia, 2 cases of superficial surgical site infection, 1 case of deep surgical site infection, 1 case of false aneurysm of a common femoral artery and 1 case of hematoma. Follow-up was performed in all patients with an average delay of 14 months [1-60]. There were 1 case of operative mortality and 2 cases of late mortality.
\end{abstract}

Keywords: Mycotic Aneurysms, Surgery, Senegal

\section{Introduction}

The term mycotic aneurysm was first used by Sir William Osler in 1885 to describe infected aneurysm that had developed as complications of bacterial endocarditis. At present, the term mycotic aneurysm is used to describe all kinds of infected aneurysms irrespective of causative pathogen [1]. Mycotic aneurysms have an incidence of 1 to $2 \%[2]$. However aortic mycotic aneurysm is associated with 
mortality rates of $15-50 \%$ [3]. Early recognition is critical to prevent poor outcome; clinical suspicion must be high, as imaging interpretation may be key to suggesting diagnosis [4]. Before the era of antibiotics, syphilis was most commonly observed. However, with the increase in arterial interventional procedures and intravenous drug use, Grampositive organisms such as Staphylococcus and Streptococcus are observed. Risk factors include interventional vascular procedures, infectious endocarditis, immunodeficiency and drug use [5]. Treatment of mycotic aneurysms involves intravenous antibiotics and most frequently surgical intervention with bypass grafts [6]. Endovascular repair, while controversial, has been used with success [7-8]. Despite available treatments, mortality is high, particularly in the case of aneurysmal rupture. Prognosis is grave when diagnosis is delayed. The objective of our study is to analyze the clinical aspects, to evaluate the results of the surgery and to compare our results with those of the literature where the management of these aneurysms remains problematic.

\section{Materials and Methods}

This was a descriptive retrospective study over a 12-years period from December 2005 to February 2018. All patients undergoing surgery for mycotic aneurysms with an archived record at the thoracic and cardiovascular surgery department of Fann University Hospital were included in the study. All patients with incomplete records were excluded from the study. The data were recorded for microsoft Excell 2013.

\section{Results}

The total number of our series was 12 cases and the average age at the time of surgery was 37 years old [17-83 years]. There was no predominance of sex with 6 male and 6 female (sex ratio 1). There was a notion of smoking in 3 cases / 12 without any case of addiction. Four of 12 cases had a known concept of heart disease with mitral insufficiency, aortic insufficiency, and complete atrioventricular block. A patient presented in his antecedents a notion of syphilis treated and declared cured. The clinical symptomatology was made of fever in 4 cases / 12, of pain in 11 cases / 12 with 3 cases of intermittent claudication. The clinical examination found a swelling with vascular characters in 11 cases / 12 with an average diameter of $9.3 \mathrm{~cm}$. There was one case of paralysis of the ulnar nerve. Two out of 12 patients had signs of acute limb ischemia. The arterial echodoppler was performed in 11 cases / 12, making the diagnosis and shows a localized aneurysm in the femoral joint ( 2 cases), popliteal ( 2 cases), superficial femoral (2 cases), subclavian (1 case), humeral ( 1 case), external iliac (1 case), common iliac (1 case) and infrarenal abdominal aorta (1 case). Echodoppler found 6 cases of false aneurysms. The angioscan was performed in 8 cases and shows 4 cases of sacciform aneurysms. The average anteroposterior diameter was 7.37 $\mathrm{cm}$ [1.74-16.9] and transverse $6.79 \mathrm{~cm}$ [0.8-12.9].

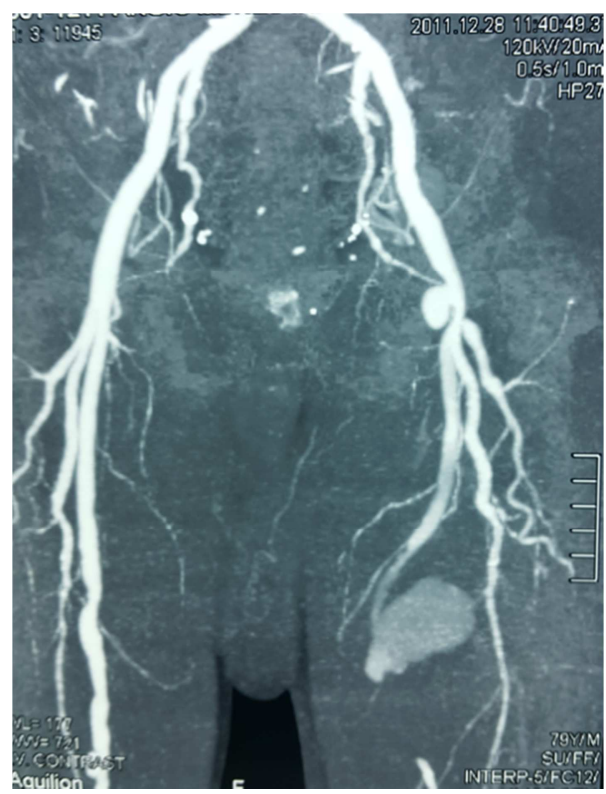

Figure 1. CT scan shows a false aneurysm in superficial femoral artery.

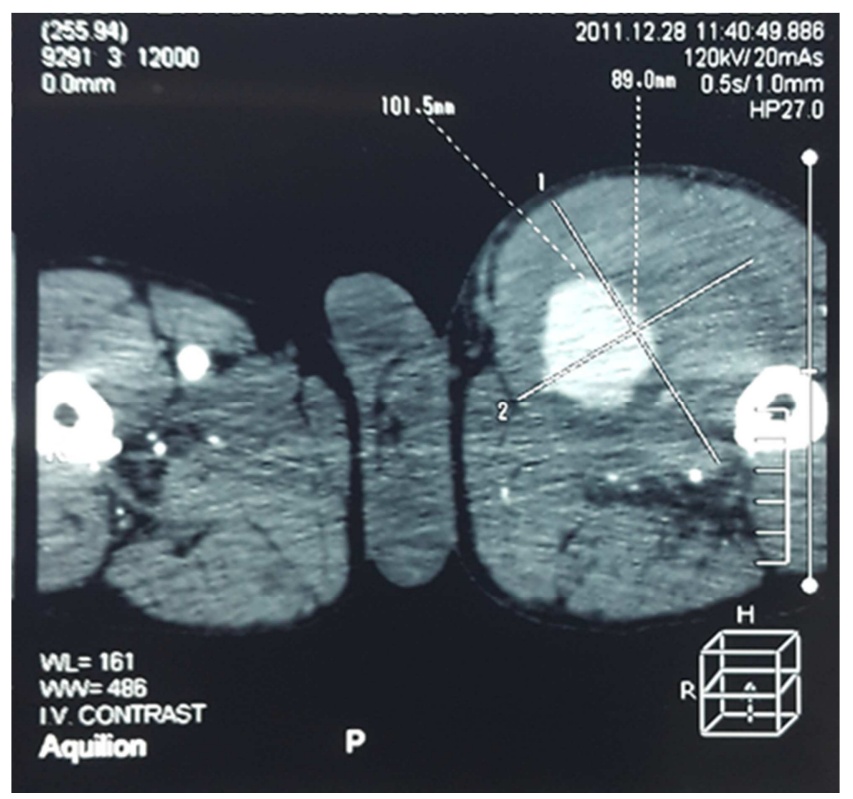

Figure 2. False aneurysm in superficial femoral artery.

There were 3 cases of compression of neighboring structures (ureter and jugular vein). Four cases of femoral artery aneurysm were found in other locations, including the infrarenal abdominal aorta, the common iliac artery and the external iliac artery. Cardiac ultrasound was performed in 8 cases and shows in 3 cases of mitral endocarditis confirmed in 1 case on transesophageal ultrasound. The biological assessment showed a C-reactive protein positive in 6 cases, the number of white blood cells was greater than 10000 elements / $\mathrm{mm} 3$ in 6 cases. HIV and syphilitic serologies were negative in all cases. All patients benefited from open surgery. The approach was a xyphopubic laparotomy (2 cases), popliteal (medial) approach (2 cases), scarpa (4 cases), elbow fold (1 case), lateral sternocervicotomy (1 case), and a tummy approach (1 case), left pararectal (1 
case). The exploration shows 9 cases of false aneurysms with signs of local infection in 5 cases.

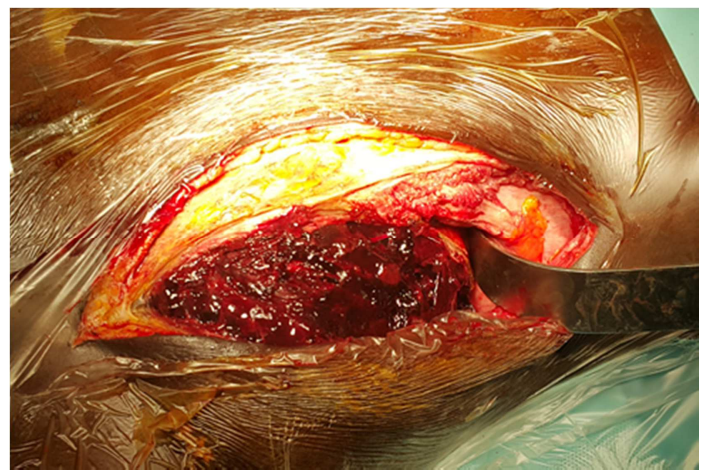

Figure 3. Operative view show an hematoma after pararectal incision.

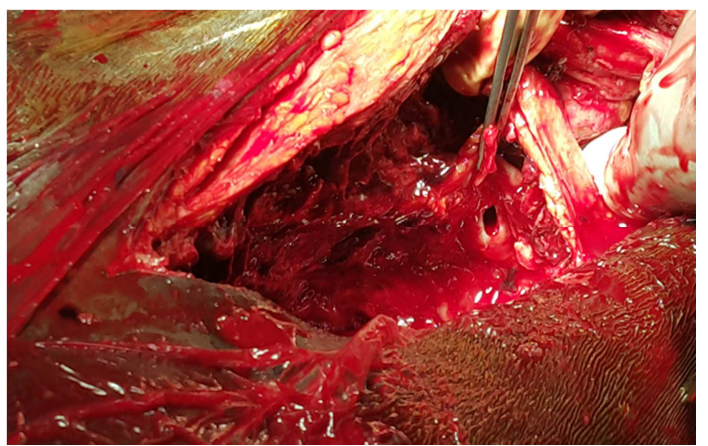

Figure 4. Operative view shows an aneurysmal collar.

Flattening with excision of the infected tissues was performed in all cases. The restoration of vascular continuity was immediate in all cases using end-to-end direct anastomosis in 8 cases, 2 cases of extra-anatomical bypass using a dacron tube and two cases of anatomical bypass using the saphenous vein in situ. Cytobacteriological examination of the collection fluid isolated from Pseudomonas aeruginosa ( 1 case) and Salmonella (1 case). A post-operative probabilistic antibiotherapy was adapted in all cases secondarily to the antibiogram for an average duration of 71 days [42-120 days]. The average duration of hospitalization was 25 days [2-60 days]. Early complications were dominated by 2 cases of acute limb ischemia, 2 cases of superficial surgical site infection, 1 case of deep surgical site infection and 1 case of false aneurysm of a common femoral artery and 1 case of hematoma. Follow-up was performed in all patients with an average delay of 14 months [1-60]. During the follow-up, a patient presented an ischemic cerebrovascular accident on a site of mitral endocarditis with a good evolution under medical treatment with physiotherapy, a case of regressive occlusive syndrome after the installation of a nasogastric tube and a case of false aneurysm of the common femoral artery requiring extraanatomical iliofemoral bypass using the contralateral saphenous vein. One amputation was performed for ischemic gangrene of the limb. There were 1 case of operative mortality, 2 cases of late mortality, one in a chronic renal failure with uremic shock and the 2 nd case in a septic shock on suppuration of an amputation stump.

\section{Comments}

Mycotic aneurysms may be present in any vascular territory, however, for peripheral locations, proximal involvement appears to be more frequent. In the Burdon series [5], out of 21 cases of mycotic aneurysms, 9 iliofemoral and 8 popliteal sites are described. These results are similar with those of our study. The pathogenic mechanisms involved in the formation of mycotic aneurysms may be septic emboli obstructing the vasa vasorum (common in bacterial endocarditis), neighboring infections reaching the arterial wall, direct bacterial inoculation or bacterial transplantation on an intimal lesion or atheroma plaque. Risk factors for mycotic aneurysms include endothelial lesions caused by atherosclerosis including the presence of aneurysm, history of infection including bacteremia, and iatrogenic arterial lesions [2]. In the literature, there are rare cases that can occur after local treatment with Bacillus Calmette Guerin in bladder cancers [9]. Although they are rare, mycotic aneurysms are a potentially severe complication of infective endocarditis that usually requires a specific therapy. In addition, as a minor criterion of the modified Duke-Li classification, detection of mycotic aneurysms affects the diagnostic certainty of infective endocarditis [10]. In our study, three patients presented signs of infectious endocarditis. The size and mobility of vegetation are strong predictors of embolization. Mycotic aneurysms are more commonly seen in the cerebral arteries than in the arteries of the limbs. Arterial complications are most often made of acute limb ischemia (20 to $30 \%$ ) and involve the lower limb more often than the upper limb [11]. The outcome of peripheral aneurysms is relatively better than of intracranial or aortic aneurysms in general $[12,13]$. Several cases reports have documented the resolution of infected intracranial aneurysms measuring less than $10 \mathrm{~mm}$ in diameter with medical treatment alone [14]; however, most peripheral aneurysms do not shrink spontaneously. In some cases reports, the authors have described rapidly increased peripheral aneurysms causing compression neuropathy or rupture [15]. The cornerstone of management is to have a strong clinical presumption and positive diagnosis [2]. The classic clinical presentation is a painful, pulsatile, bulky swelling in a patient with general signs such as fever. The diagnosis is mostly based on imaging. CT angiography is the most efficient procedure for diagnosis [16]. Magnetic Resonance Imaging can be an alternative when contrast injection is contraindicated. A saccular aneurysm, rapid enlargement of the original aneurysm and perianeurismal soft tissue mass have been reported in the radiological manifestations typical of mycotic aneurysms [2, 17]. To determine whether patients with aortic mycotic aneurysms had difference presenting features and outcomes compared with patients with non aortic mycotic aneurysms including iliac and visceral aneurysms. These patients group were 
compared in the Deipolyi's study. Clinical presentation was similar, though non aortic aneurysms were presenting more frequently fevers than aortic aneurysms. Imaging findings were also similar, though aortic aneurysms tended to be more often a saccular outpouching, likely due to the larger size of the involved vessel; also, aortic aneurysms tended to be associated more frequently with rupture, though this finding did not reach significance [18]. Management options include open surgery and endovascular treatment with long-term antibiotic therapy [2, 17]. Open surgery remains one of the best options. Arterial ligation with aneurysm resection and local debridement with excision of all necrotic tissue and drainage remains the first stage of treatment; Revascularization at the same time offers a better functional prognosis of the limb and the vein is more resistant to infection [19]. In some series, the prosthesis is covered by a plasty with the large omentum associated with a local instillation of antibiotics [9]. Endovascular treatment is recommended especially if there is a high surgical risk and has the advantage of being minimally invasive [2]. In our series, all the patients have benefited from open surgery. The restoration of vascular continuity was immediate in all cases by direct anastomosis in 5 cases, extra-anatomic bypass in 2 cases and anatomical in 1 case. Another valuable treatment option is the hybrid approach combining endovascular treatment with open surgery. This method consists in urgently placing a stent at the level of the arterial defect under local anesthesia followed by debridement of the infected tissues at a later date [19]. After surgery, broad-spectrum antibiotic therapy covering Gramnegative and anaerobic germs should be started. Some studies recommend 4-6 weeks of parenteral antibiotic therapy $[20,21]$. Muller thinks antibiotics should be given for at least 3 months and should be stopped only if the signs of infection are gone [4]. Mycotic aneurysms are associated with high mortality and morbidity. In Chen's Study hospital mortality was $11.8 \%$ [1]. In the Deipolyi series, mortality at 6 months was $19 \%$ despite surgical or endovascular treatment [18].

\section{Conclusions}

Mycotic aneurysms are rare but potentially serious because they can be life-threatening and functional. Despite the advent of endovascular treatment, open surgery still holds its place. In some cases the hybrid approach seems to be the best solution. All these techniques are covered by an antibiotherapy adapted to the antibiogram whose duration depend on the institution. The long-term results are satisfactory in terms of survival and quality of life as evidenced by our results but especially better for peripheral aneurysms. Early diagnosis remains the best way for optimal management and improvement of results and this requires a strong clinical presumption in front of a painful, pulsating and voluminous swelling in a patient with general signs such as fever.

\section{References}

[1] Chen IM, Chang H., Hsu C., Lai S., Shih C. Ten-Year Experience with Surgical Repair of Mycotic Aortic Aneurysms. J Chin Med Assoc 2005; 68(6):265-271.

[2] Kota A. A, Sen I., Dheepak Selvaraj A., Premkumar P., Ponraj S., Agarwal S. Mycotic aneurysm case serie. Indian Journal of vascular and endovascular surgery, Jan-Mars 2015, Issue 1, Vol 2, Page 38-40.

[3] Kyriakides C., Kan Y., Kerle M., Cheshire NJ., Mansfield AO., Wolfe JH. 11 Years experience with anatomical and extraanatomical repair of mycotic aortic aneurysms. Eur J Vasc Endovasc Surg 2004; 27:585-9.

[4] Muller BT., Wegener OR., Grabitz K., Pillny M., Thomas I., Sandmann W. Mycotic aneurysms of the thoracic and abdominal aorta and iliac arteries: experience with anatomic and extraantomic repair in 33 cases. J Vasc Surg 2001;33:106-13.

[5] Burdon J. R, Ravat F., Desthieux J. M, Descotes J. Les anévrismes mycotiques des membres inférieurs . A propos de 21 cas. Chirurgie, 1988, $114: 482-9$.

[6] Oderich GS, Panneton JM, Bower TC, Cherry KJ, Rowland CM, Noel AA et al. Infected aortic aneurysms: aggressive presentation, complicated early outcome, but durable results. J Vasc Surg. 2001;34:900-8.

[7] Jorna FH, Verhoeven EL, Bos WT, Prins TR, Dol JA, Reijnen MM. Treatment of a ruptured thoracoabdominal aneurysm with a stent-graft covering the celiac axis. J Endovasc Ther 2006;13:770-4.

[8] Stanley BM, Semmens JB, Lawrence-Brown MM, Denton M, Grosser D. Endoluminal repair of mycotic thoracic aneurysms. J Endovasc Ther 2003;10:511-5.

[9] Roeke T., Hovsibian S., Schlejen P., Dinant S., Koster T., Waasdorp E. A mycotic aneurysm of the abdominal aorta caused by Mycobacterium bovis after intravesical instillation with bacillus Calmette-Guérin. J Vasc Surg Cases and Innovative Techniques 2018; 4:122-5.

[10] Mikail N., Benali K., Ou P., Slama J., Hyafil F., Le Guludec D., Rouzet F. Detection of Mycotic Aneurysms of Lower Limbs By Whole-Body F-FDG-PET. JACC: Cardiovascular Imaging. Vol 8;n 7, 2015 July:859-62.

[11] Pessinaba S., Kane Ad., Ndiaye M. B., et al. Vascular complications of infective endocarditis. Médecine et Maladies $\begin{array}{llll}\text { infectieuses } & 42 & (2012) & 213-217,\end{array}$ http//dx.doi.org/10.1016/j.medmal.2012.03.001.

[12] Peters PJ, Harrisson T, Lennox JLA dangerous dilemma: management of infectious intracranial aneurysms complicating endocarditis. Lancet Infect Dis 6: 742-748, 2006.

[13] Sonneville R, Mirabel M, Hajage D. et al. Neurologic complications and outcomes of infective endocarditis in critically ill patients: The endocardite en reanimation prospective multicenter study. Crit Care Med 39: 1474-1481, 2011.

[14] Leon LR, Psalms SB, Labropoulos N, Mills JL. Infected upper extremity aneurysms: a review. Eur J Vasc Endovasc Surg 35:320-331, 2008. 
[15] Kang GC, Wong CH, Lee JY. Simultaneous infected pseudoaneurysm and suppurative tenosynovitis resulting from radial artery cannulation. Surg Infect (Larchmt) 9:489-492, 2008.

[16] Deser S. B., Demirag M. K. Infective endocarditis and incidental popliteal artery mycotic aneurysm. Cor and Vasa 59 (2017) e291-e293.

[17] Polytarchou K., et al. Ulnar pseudoaneurysm complicating infective endocarditis. The role of endovascular stenting. Hellenic Journal of Cardiology (2017), https://doi.org/10.1016/j.hjc.2017.11.007.

[18] Deipolyi AR., Bailin A., Khademhosseini A., Oklu R. Imaging
Findings, Diagnosis and Clinical Outcomes in Patients With Mycotic Aneurysms: Single Center Experience. Clinical Imaging 40(2016) 512-516.

[19] Karkos C. D, Kalogirou T. E, Giagtzidis I. T, Papazoglou K. O. Ruptured Mycotic common femoral artery pseudoaneurysm. Tex Heart Inst J 2014;41(6):634-7.

[20] Mani K., Bjork M., Lunkvist J., Wanhaine A. Improved long term survival after abdominal aortic aneurysm repair. Circulation 2009; 120:201-11.

[21] Moneta G. L, Taylor L. M. Jr, Yeager R. A, and al. Surgical treatment of infected aortic aneurysm. Am J Surg 1998; 175:396-9. 